\title{
Research Article \\ Research on 5G-Oriented Wireless Sensor Array of Millimeter Hybrid Beam Sensing Terminal
}

\author{
Shengli Yan \\ School of Automobile and Mechatronics Engineering, Zhoukou Vocational and Technical College, Zhoukou, Henan 466000, China \\ Correspondence should be addressed to Shengli Yan; 2001050012@zkvtc.edu.cn
}

Received 19 August 2021; Accepted 30 August 2021; Published 20 September 2021

Academic Editor: Guolong Shi

Copyright ( 2021 Shengli Yan. This is an open access article distributed under the Creative Commons Attribution License, which permits unrestricted use, distribution, and reproduction in any medium, provided the original work is properly cited.

\begin{abstract}
With the rapid development of information technology, facing the problems and new challenges brought by mobile Internet and Internet of things technology, as one of the key technologies of 5G, millimeter-wave mobile communication $(28 / 38 / 60 / 70 \mathrm{GHz})$ which can realize gigabit $(\mathrm{GB} / \mathrm{s}$, or even higher) data transmission rate has also attracted extensive attention of wireless researchers all over the world, it has quickly become a research hotspot in the field of wireless communication. In the millimeter-wave massive MIMO downlink wireless sensor system, a block diagonal beamforming algorithm based on the approximate inverse of Neumann series is improved to obtain complete digital beamforming. Then, when designing hybrid beamforming, channel estimation and high-dimensional singular value decomposition are required for traditional analog and digital hybrid beamforming. A low complexity hybrid beamforming scheme is designed. An improved gradient projection algorithm is proposed in the design of analog beamforming, which can solve the problem of high computational complexity and less damage to guarantee and rate. Simulation results show that the hybrid beam terminal of the sensor reduces the number of RF links required for full digital beamforming and is as close to the spectral efficiency performance of full digital beamforming as possible. The results show that the performance of the designed hybrid beamforming scheme can still be close to that of the pure digital beamforming scheme without involving channel estimation and SVD decomposition.
\end{abstract}

\section{Introduction}

With the development of mobile multimedia applications, the improvement of end-user requirements, and the popularity of devices, the demand of mobile communication services for system data transmission rate and transmission bandwidth is increasing day by day. On the one hand, the current 100 megabit data rate provided by traditional cellular mobile networks and wireless LAN has been difficult to meet the demand for higher rate of fast-growing application services. In addition, with the development of mobile communication technology and Internet of things technology, the number of global mobile communication network equipment and Internet of things equipment connections will also increase exponentially in the future, reaching a scale of 100 billion [1]. On the other hand, at present, most of the spectrum resources in the low-frequency band (below $5 \mathrm{GHz}$ ) have been occupied by many mobile communication ser- vices, including wireless LAN, multimedia communication, satellite navigation communication, broadband mobile communication, and wireless positioning services, which makes the available and valuable spectrum resources increasingly exhausted. Therefore, in order to meet the needs of future mobile data growth, the fifth-generation mobile communication $5 \mathrm{G}$ system came into being. Researchers said that in order to finally realize the $5 \mathrm{G}$ vision of "information comes at will, everything touches and," 5G system needs to have higher performance compared with $4 \mathrm{G}$ system, and millimeter-wave communication is one of the key technologies of the fifth-generation mobile communication technology (5G), which has attracted extensive research and attention. Because the millimeter-wave band has the characteristics of high path loss and low penetration, millimeterwave communication needs to combine large-scale multiple input multiple output (MIMO) technology [2], use largescale antenna array, and obtain strong gain through 
beamforming technology to resist high path loss and signal attenuation. All digital beamforming requires the system to provide the same number of RF links as the number of antennas, which brings high cost and high power consumption. In order to solve this problem, a hybrid beamforming technology is proposed, which combines digital domain and analog domain beamforming. Hybrid beamforming combined with analog beamforming and baseband digital beamforming can reduce the number of RF links required for full digital beamforming and approach the spectral efficiency performance of full digital beamforming as much as possible, so it can effectively reduce the number of RF links and reduce the complexity of the system.

At present, although there are many researches on hybrid beamforming technology, it is still an open problem to design the best hybrid beamforming algorithm suitable for millimeter-wave communication [3]. The biggest disadvantage of millimeter wave is its very high propagation loss. Due to the characteristics of the high-frequency wavelength end, the propagation loss of millimeter wave in free space is several $\mathrm{dB}$ more than that in the low-frequency band (2.4 GHz and $5 \mathrm{GHz}$ band) currently used (the propagation loss in free space is directly proportional to the frequency and inversely proportional to the wavelength). Therefore, it is necessary to introduce beamforming technology and use antenna array at the transmitter and receiver to obtain additional directional antenna gain. For the same size antenna, the gain of directional antenna is much higher than that of omnidirectional antenna used in a low-frequency communication system [4]. Therefore, the beamforming of directional antenna can be used to compensate the excessive free space propagation loss in millimeter-wave communication. In addition, in the high-frequency band, millimeter-wave propagation characteristics will also be affected by rain and gas. The higher the rainfall rate, the greater the millimeterwave propagation loss. At the same time, due to the gas absorption in the atmosphere, if the transmission distance exceeds tens of meters, it will lead to considerable attenuation at some frequencies of millimeter wave, such as $60 \mathrm{GHz}$. In the millimeter-wave wireless communication system, it is easy to configure and use more antenna units, and the increase in the number of antennas means that the traditional digital beamforming technology is still used, which becomes unacceptable in cost and complexity [5].

Aiming at the above problems, focusing on the low complexity partial connection architecture, this paper studies the relationship between the number of RF links and energy efficiency and spectral efficiency, focusing on the optimal number of RF links on the premise of ensuring the gain. Firstly, this paper analyzes the relationship between energy efficiency, spectral efficiency, and the number of RF links under the partial connection architecture and then proves that there is an optimal power point, namely, green point, on the premise of considering the power of link devices. Secondly, the influence of link number on energy efficiency under the condition of given spectral efficiency is analyzed. Focusing on the beam training process of IEEE802.11ad standard, the sector level scanning and beam optimization process used in a millimeter-wave communication system are described in detail, and the antenna beam state at the sensing end in each stage is analyzed. Then, the link-level simulation of IEEE802.11ad is carried out, the wireless sensor simulation platform is built, and the simulation curve is given for calibration. Aiming at the problem that the feedback overhead of millimeter hybrid beamforming receiver is too large, a millimeter hybrid beamforming training feedback design scheme suitable for IEEE802.11ad is proposed to effectively meet the beamforming training requirements of the millimeter-wave communication system. The analysis shows that when the spectral efficiency is determined, there is an optimal number of RF links, which can make the system obtain the best energy efficiency. Finally, the relationship between the optimal energy efficiency and the number of RF links is analyzed. The analysis results show that when the number of RF links is not related to the number of antennas in each subarray, the optimal energy efficiency increases monotonically with the number of RF links.

\section{Related Works}

Recently, hybrid beamforming technology in millimeterwave communication has been widely studied. Kim et al., the wireless communication research center of Samsung company, proposed a hybrid beamforming algorithm based on the idea of equivalent channel [6]. The idea of the algorithm is to select the optimal analog matrix through codebook search according to the criterion of optimal capacity to form a low-dimensional equivalent channel matrix, and then, according to the equivalent channel matrix, the common linear precoding algorithm is used to solve the digital beamforming matrix. Based on the idea of equivalent channel, Samsung and Huawei American research center further proposed a multiuser MIMO hybrid beamforming strategy [7]. This strategy is based on RF beam control vector and realizes analog beamforming through beam search, which has high implementation complexity. Subsequently, Liang of the University of Victoria proposed a low complexity multiuser hybrid beamforming algorithm directly based on channel state information [8]. The algorithm obtains the analog beamforming matrix by phase extraction of channel state information and then performs low-dimensional digital beamforming at the baseband based on the equivalent channel idea. Another classical hybrid beamforming algorithm is proposed by Omar EI Ayach of the University of Texas. This kind of algorithm is a spatial sparse hybrid beamforming algorithm based on signal recovery [9]. It fully analyzes and makes use of the sparse characteristics of millimeter wave. According to the criterion of optimal capacity, the original nonconvex optimization problem is transformed into a suboptimal convex optimization problem through matrix decomposition operation. A feasible optimization algorithm orthogonal matching pursuit (OMP) algorithm is provided to solve the digital beamforming matrix and ana$\log$ beamforming matrix based on the idea of signal recovery. Singh and Ramakrishna further proposed a low complexity hybrid beamforming algorithm based on a feasible codebook [10]. The algorithm uses the sparse characteristics of millimeter-wave channel to concentrate the 
codebook space of analog beamforming into the dominant direction beam set determined by channel departure angle (AOD) and arrival angle (AOA), reducing the cardinality of search space. At the same time, a low complexity algorithm for estimating the angle of arrival of the receiver by using the correlation of the received subarray signals is also presented.

The basic idea of hybrid beamforming is as follows: at the antenna end, based on the characteristics of millimeter wave length, using large-scale antenna array and directional beam can not only reduce the interference between users and channels but also increase the signal strength and resist attenuation. At present, the antenna technology in the lowfrequency band generally realizes beamforming in the digital domain. In the millimeter-wave band, due to the increase of the number of antennas, all digital beamforming cannot be realized. Therefore, millimeter-wave beamforming is carried out in both the digital domain and the analog domain. Generally, analog beamforming (ABF) is realized at the antenna array, and digital beamforming (DBF) is implemented in the baseband. Such beamforming technology is called millimeter-wave hybrid beamforming (HBF) technology. Reference [11] considered the hybrid beamforming design in a broadband system with orthogonal frequency division multiplexing and transformed the analog beamforming design of frequency selective channel into that of flat fading channel. Reference [12] uses the alternating minimization algorithm based on coordinate descent to obtain mixed digital and analog beamforming and proves that it can obtain the optimal performance under low-resolution phase shifter. Under the multiuser channel model, Reference [13] uses a hierarchical codebook training method to design hybrid beamforming. However, the size of the codebook always increases linearly or even exponentially with the number of antennas, resulting in the complexity of analog beam search. In Reference [14], we designed a two-layer alternating optimized spectrum sharing framework based on the interference alignment (IA) method. Reference [15] studies the hybrid analog and digital beamforming design of the multiuser millimeter-wave communication system based on the minimum mean square error standard. In Reference [16], the penalty double decomposition method is used to solve the nonconvex hybrid beamforming problem of the millimeter-wave multiuser MIMO system, which can ensure the convergence of the PDD method. In Reference [17], considering the optimal user combination and power allocation, the nonconvex constraint problem for hybrid beamforming is transformed into a convex positive semidefinite problem. The focus of the above research is to maximize the system spectral efficiency in the design of hybrid beamforming in millimeter-wave communication, but little attention is paid to how to reduce the computational complexity of the algorithm in the design of hybrid beamforming. In the millimeter-wave massive MIMO downlink communication system, a block diagonal beamforming algorithm based on the approximate inverse of Neumann series is improved to obtain complete digital beamforming, and then, the alternating minimization algorithm is used as the main design principle to determine the hybrid digital beamforming and
TABLE 1: Simulation parameters.

\begin{tabular}{lccccc}
\hline$P_{\text {rf_circuit }}$ & $P_{0}$ & $W$ & $N_{0}$ & $\eta_{\mathrm{PA}}$ & Channel gain \\
\hline $1 \mathrm{~W}$ & $1 \mathrm{~W}$ & $6 \times 10^{10} \mathrm{~Hz}$ & $10^{-17} \mathrm{dBm} / \mathrm{Hz}$ & 0.375 & $-100 \mathrm{~dB}$ \\
\hline
\end{tabular}

analog beamforming. In the design of analog beamforming, an improved gradient projection algorithm is proposed, which can solve the problem of high computational complexity and less damage to guarantee and rate.

\section{Millimeter Hybrid Beam Sensor Terminal Array Architecture}

3.1. Hybrid Beam Sensor Terminal Wireless Channel Model. Beamforming is to weight the signals on multiple antenna reduction elements by feeding and then combine them to form the required highly directional signals. From the perspective of antenna pattern, sample transportation is equivalent to forming a directional beam and simulation parameters was shown in Table 1. In practice, digital beamforming is used for beamforming [18], and each antenna is connected with a radio frequency chain ( $\mathrm{RF}$ chain), including a mixer, power amplifier (PA), and phase shifter. The amplitude and phase of the signal are controlled in the digital domain after the signal is converted into a digital signal by an A/D converter. Moreover, due to the limitation of antenna size and other factors, the number of antennas is small, and there are not too many antennas on the equipment, so the cost and overhead are not very large, as shown in Figure 1.

Due to the high path loss characteristics of millimeterwave signals and the limited scattering characteristics of millimeter-wave channels, existing studies [19] have used geometric channel models to model millimeter-wave channels. Under this model, based on the unified linear array (ULA), the discrete-time narrowband channel matrix $H$ in the system can be expressed as

$$
H=\sum_{i=1}^{N_{\mathrm{cl}}} \sum_{l=1}^{N_{\mathrm{ray}}} \gamma \alpha_{i} a_{t}{ }^{H} a_{r}\left(\phi_{i}\right)\left(\theta_{i, l}\right)
$$

where $N_{\mathrm{cl}}$ and $N_{\text {ray }}$ represent the number of scattering clusters in the channel and the number of propagation multipaths in the cluster, respectively, $\alpha_{i, l}, \phi_{i, l} \in\left[\phi_{i, \min }, \phi_{i, \max }\right]$, and $\theta_{i, l} \in\left[\theta_{i, \min }, \theta_{i, \max }\right]$ represents the complex gain power value, angle of arrival (AOA), and AOD angle of the $l$ th multipath in the $i$ th scattering cluster of the channel, respectively, where $\phi_{i, l} \in\left[\phi_{i, \text { min }}, \phi_{i, \text { max }}\right]$ and $\theta_{i, l} \in\left[\theta_{i, \text { min }}, \theta_{i, \text { max }}\right]$ represent the angular diffusion interval of $\mathrm{AOA}$ and $\mathrm{AOD}$ angles of the $i$ th scattering cluster, respectively, and $E_{\left|\alpha_{i, l}\right|^{2}}$ $=\sigma_{i, \alpha}^{2} \cdot \gamma$ is a constant so that constraint $E_{\|H\|_{F}^{2}}=N_{t} N_{r}$ holds and satisfies $\gamma=\left(N_{t} N_{r}\right)^{1 / 2}\left(N_{\text {ray }} \sum_{i=1}^{N_{c l}} \sigma_{i, \alpha}^{2}\right)^{-1 / 2}$. Finally, $a_{t}(\theta)$ and $a_{r}(\phi)$ represent the antenna array response vectors of 


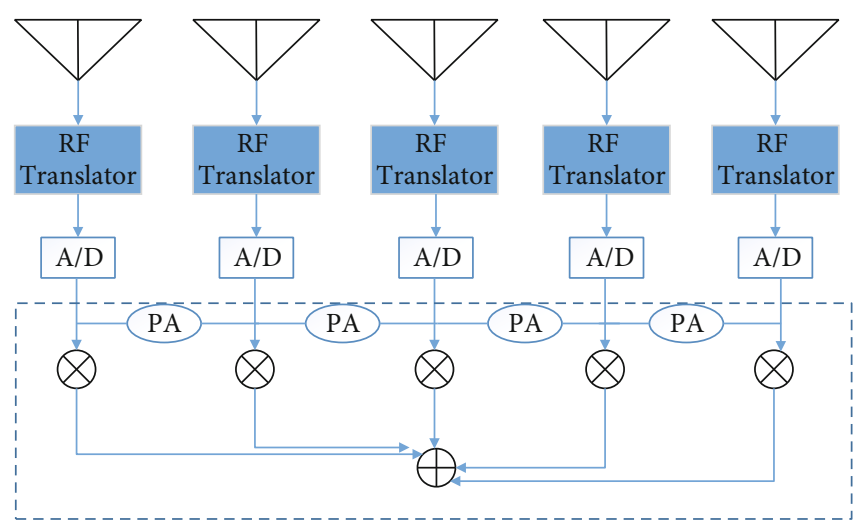

Digital beamformer

FIgURE 1: Schematic diagram of digital beamforming receiver.

BS end and end user, respectively, and $a_{t}(\theta)$ and $a_{r}(\phi)$ can be expressed as

$$
\begin{aligned}
& a_{t}(\theta)=N_{t}^{-1 / 2}\left[1, \cdots e^{i k d\left(N_{t}-1\right) \sin \theta}\right]^{T}, \\
& a_{r}(\phi)=N_{r}^{-1 / 2}\left[1, \cdots e^{i k d\left(N_{r}-1\right) \sin \theta}\right]^{T},
\end{aligned}
$$

where $k=2 \pi / \lambda$ and $d=\lambda / 2$ represent the spacing between antenna array elements and $\lambda$ indicates the wavelength of the signal.

Therefore, this paper also uses this channel model to model the channel of each user, and the user channel matrix $h$ obtained by rewriting equation (1) can be expressed as

$$
h=\sum_{i=1}^{N_{\mathrm{cl}}} \sum_{l=1}^{N_{\mathrm{ray}}} \gamma \alpha_{i, l}^{2} a_{t}^{H}\left(\theta_{i, l}\right) .
$$

In addition, during channel modeling based on the scenarios considered in this chapter, in order to better distinguish the primary cluster and secondary cluster space of each user, it is assumed that the complex gain change power of the primary cluster is much greater than that of all other secondary clusters, that is, to meet the following requirements:

$$
\sigma_{i, a}>>\sigma_{j, a}\left(\forall j \in\left\{1, \cdots N_{\mathrm{cl}}\right\}\right) .
$$

3.2. Planar Array of Wireless Sensors. Planar array refers to the arrangement of multiple array elements in the form of rectangular grid to form a planar array [20]. In the rectangular coordinate system, the number of elements on the $x$-axis is defined as $M$ and the number of elements on the $y$-axis is defined as $N$ to obtain an $M \times N$-element two-dimensional rectangular plane array. Compared with linear array, planar array has a more control factor because it is a twodimensional antenna array, which makes it have more symmetrical pattern and lower side lobe. The schematic diagram of a planar array is shown in Figure 2.
Suppose the spacing $d_{x}$ of $M$ array elements and its array function $f_{\alpha}(\theta, \varphi)$. As shown in

$$
f_{a x}(\theta, \varphi)=\sum I_{m l} \exp j(m-1)\left(k d_{x} \sin \theta \cos \varphi+\xi_{x}\right),
$$

where $I$ is the amplitude of the current on the $x$-axis and is the initial phase of the current. Similarly, we can get

$$
f_{a y}(\theta, \varphi)=\sum I_{n} \exp j(n-1)\left(k d_{y} \sin \theta \sin \varphi+\xi_{y}\right) .
$$

The matrix function of the $M \times N$-element planar array is shown in

$$
f_{a}=\sum_{m=1}^{M} I_{m} \exp j(m-1) \psi_{x} \sum_{n=1}^{N} I_{n} \exp j(n-1) \psi_{y},
$$

where $\psi_{x}=k d_{x}(\sin \theta \cos \varphi)+\xi_{x}$ and $\psi_{y}=k d_{y}(\sin \theta$ $\cos \varphi)+\xi_{y}$.

The above formula shows that the matrix function of a two-dimensional rectangular plane array is the product of two one-dimensional matrix functions along the $x$-axis and $y$-axis. If the amplitude of the current on the $y$-axis is proportional to the amplitude of the current on the $x$-axis, the current amplitude of the $(m, n)$-th antenna element on the planar array can be expressed as

$$
I_{m n}=I_{m 1} I_{1 n}
$$

It is further assumed that the amplitudes of the currents of all elements in the array are equal $\left(I_{m n}=I_{0}\right)$, so the normalized array function is

$$
F(\theta, \varphi)=\frac{\sin \left((M / 2) \psi_{x}\right)}{M \cdot \sin \left(\psi_{x} / 2\right)} \cdot \frac{\sin \left((N / 2) \psi_{y}\right)}{N \cdot \sin \left(\psi_{y} / 2\right)},
$$

where $\psi_{x}=k d_{x}(\sin \theta \cos \varphi)+\xi_{x}$ and $\psi_{y}=k d_{y}(\sin \theta$ $\cos \varphi)+\xi_{y}$

The cross-sectional direction of the plane array is shown in Figure 3. 


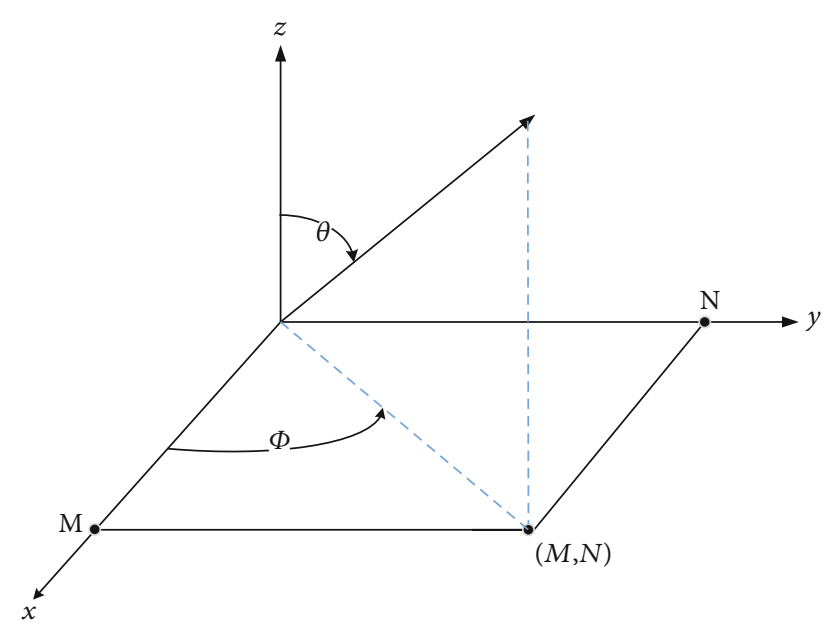

FIgURE 2: Schematic diagram of planar array antenna.

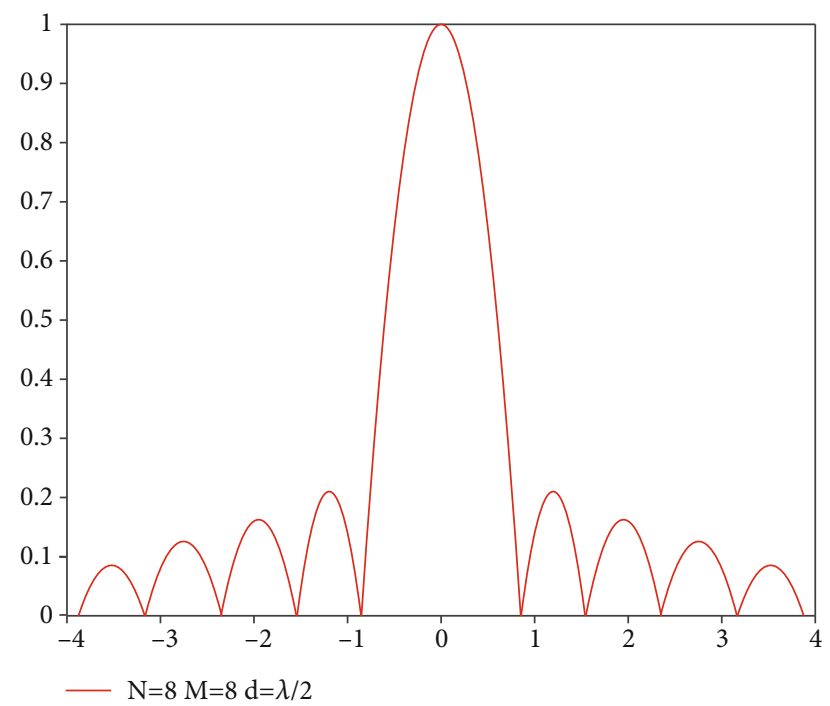

Figure 3: Section direction of plane array.

3.3. Millimeter Hybrid Beamforming System Architecture. Assuming that the number of data streams, radio frequency (RF) links, and antennas are $N$ and $N M$, in order to cascade digital beamforming and analog beamforming, Figure 4 shows the architecture of two hybrid beamforming systems. There are $N$ RF links and $N M$ antennas in each figure. The difference between the two architectures is that in architecture (a), each RF link is connected to all NM antennas, which is a fully connected architecture. All data is first digitally beamformed, then processed by each RF link, and then transmitted using all antenna elements. Each RF link in architecture (b) is only connected with $M$ antennas and only partially connected. Multistream data is first digitally beamformed and then transmitted to the analog beamforming module through a dedicated RF link, and then, $M$ antennas are used to form a subantenna array to transmit signals. Obviously, the full connection architecture is more complex than the partial connection architecture, because the full connection architecture requires each $\mathrm{RF}$ link to be connected with all antennas, and the signal must go through $N^{2} M$ signal processing paths, while there are only $N M$ partial connections. However, the beamforming gain that can be achieved in partial connection architecture is small [21], which is $1 / N$ less than that can be obtained in full connection architecture. Smart antenna belongs to array antenna, which is composed of multiple identical omnidirectional antennas. The array structure can take many forms, and the two-dimensional array is used in this paper. Because the purpose of this paper is to reduce the number of digital links on the premise of ensuring the gain, this paper focuses on some connection architectures.

Suppose there are $N$ downlink users, and each user has only a single antenna. Then, the received signal can be expressed as $y=H A D s+n$. Where $H$ is the downlink channel, we assume that there are $N$ downlink users and the number of antennas is $N M$, so the dimension of downlink channel is $N \times N M, A$ is the analog beamforming matrix, and the dimension is $N \times N M$. D is the digital beamforming matrix, and the dimension is $N \times N$; $s$ is the data of $N$ users, and the dimension is $N \times 1 ; N$ is the noise vector. As shown in Figure 4(a), the signal is transmitted to the NM antenna after passing through the digital beamforming matrix $D$ and the analog beamforming matrix $A$ in the baseband. Column $i$ in matrix $A$ is the weighting of the NM antenna to the signal on the $i$ th RF link. For the partial connection architecture in Figure 4(b), the signal on each RF link can only be transmitted to $M$ antennas. Therefore, the form of analog beamforming matrix $A$ is different from the full connection architecture, as shown in

$$
Y=\left[\begin{array}{c}
H_{1} \\
H_{2} \\
\vdots \\
H_{N}
\end{array}\right]\left[\begin{array}{ccc}
A_{1} & \cdots & 0 \\
\vdots & \ddots & \vdots \\
0 & \cdots & A_{N}
\end{array}\right] D\left[\begin{array}{c}
S_{1} \\
S_{2} \\
\vdots \\
S_{N}
\end{array}\right]+n
$$

where $H$ is the downlink channel; $H_{i}$ is the channel corresponding to the $i$ th user, and the dimension is $1 \times N M$; $A$ is the analog beamforming matrix; $a$ represents the analog beamforming matrix on the $i$ th RF link, and the dimension is $M \times 1$.

3.4. Sensing Terminal Energy Efficiency and Spectrum Efficiency. Referring to the partial connection architecture in Figure 4(b), assuming that there are $N$ users, the $M$ antennas on each subarray point to a user and ensure that there is no interference before $N$ users; the sum of the capacity of $N$ users can be expressed as

$$
C=2 N \cdot W \log _{2} M \eta_{\mathrm{PA}} P W^{-1} N_{0}^{-1},
$$

where $W$ is the bandwidth, $P$ is the total power of power amplifiers (PA) on a subarray, $\eta_{\mathrm{PA}}$ is the power amplifier efficiency, and $N_{0}$ is the noise density. Assuming 


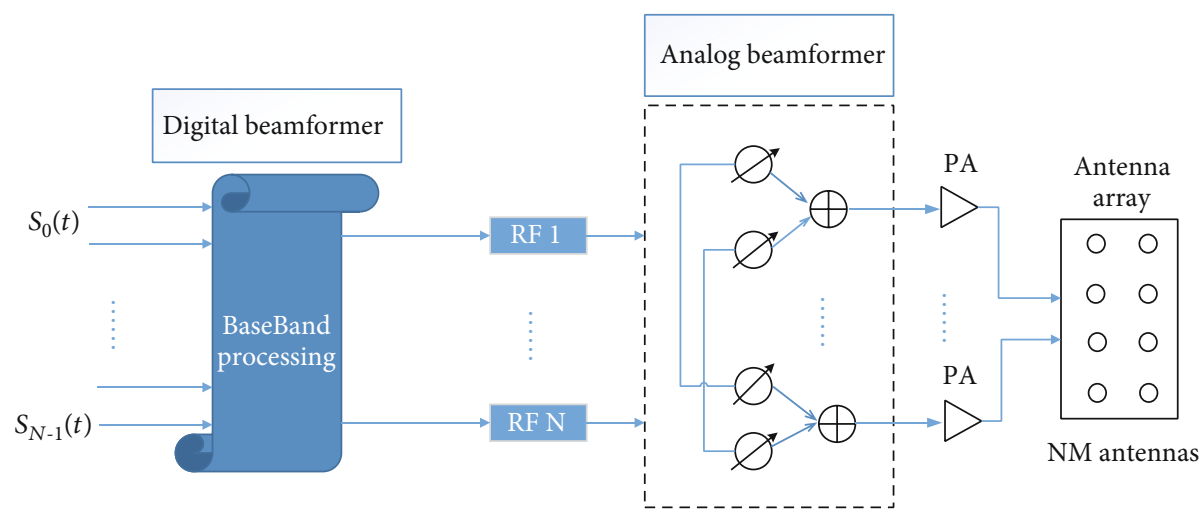

(a)

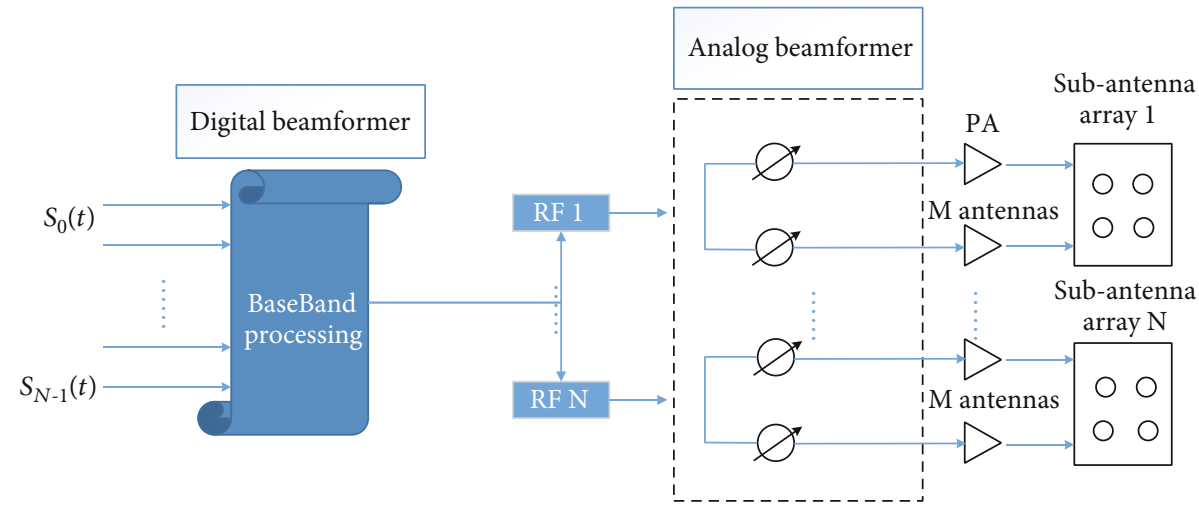

(b)

FIgURE 4: Two hybrid beamforming architectures: (a) full connection; (b) partial architecture.

that the channel gain is 1 , the spectral efficiency (SE) of the architecture is

$$
\eta_{\mathrm{SE}}=C \cdot W^{-1}=2 N \log _{2} M \eta_{\mathrm{PA}} P W^{-1} N_{0}^{-1}
$$

Accurate power models are needed to calculate energy efficiency, but in actual use, different base stations have different types and are usually produced by different suppliers. Therefore, the simplified power model adopted in this paper is

$$
P_{\text {total }}=N P_{0}+P_{\text {common }}+N M P_{\text {rf_circuit }},
$$

where $P_{\text {total }}$ is the total power, NP is the power of $N$ RF links, and $P_{\text {static }}$ is the basic power for normal operation of the equipment, including the basic power $N P_{0}$ describing $N$ RF links, the basic power $P_{\text {common }}$ independent of the number of links, and the circuit power $N M P_{\text {rf_circuit }}$ related to the total number of antennas NM.

Therefore, the relationship between energy efficiency (EE) and spectral efficiency is

$$
\begin{aligned}
\eta_{\mathrm{EE}}= & C \cdot P_{\text {total }}^{-1}=\eta_{\mathrm{SE}}\left\{\frac{N_{0} N}{M \eta_{\mathrm{PA}}}\left(2^{\eta_{\mathrm{SE}}} / N-1\right)\right. \\
& \left.+W^{-1}\left(N P_{0}+P_{\text {common }}+N M P_{\text {rf_circuit }}\right)\right\}^{-1} .
\end{aligned}
$$

In the hybrid beamforming architecture, in order to save power as much as possible, we hope to find the best working point of the system. The best working point can be found on the EE-SE curve, also known as the green point on the EE-SE curve. The basic idea is to optimize the system parameters so that the maximum energy efficiency (EE) can be obtained within the specified spectral efficiency (SE) range.

\section{Experiment and Analysis}

4.1. Signal-to-Interference Noise Ratio and Spectral Efficiency. This scheme is mainly evaluated by signal-tointerference plus noise ratio (SINR) and spectral efficiency (SE). After hybrid beamforming, the medium-term expected user power of the output signal is

$$
P_{\text {expectation }}=\left|\left(F_{A B F} W_{D B F}\right)^{H} a\left(\theta_{1}\right) s_{1}(n)\right| .
$$

The interfering user power in the output signal is

$$
P_{\text {interfere }}=\left|\left(F_{A B F} W_{D B F}\right)^{H} n(n)\right| .
$$

The noise power in the output signal is

$$
P_{\text {noise }}=\left|\left(F_{A B F} W_{D B F}\right)^{H} n(n)\right| \text {. }
$$




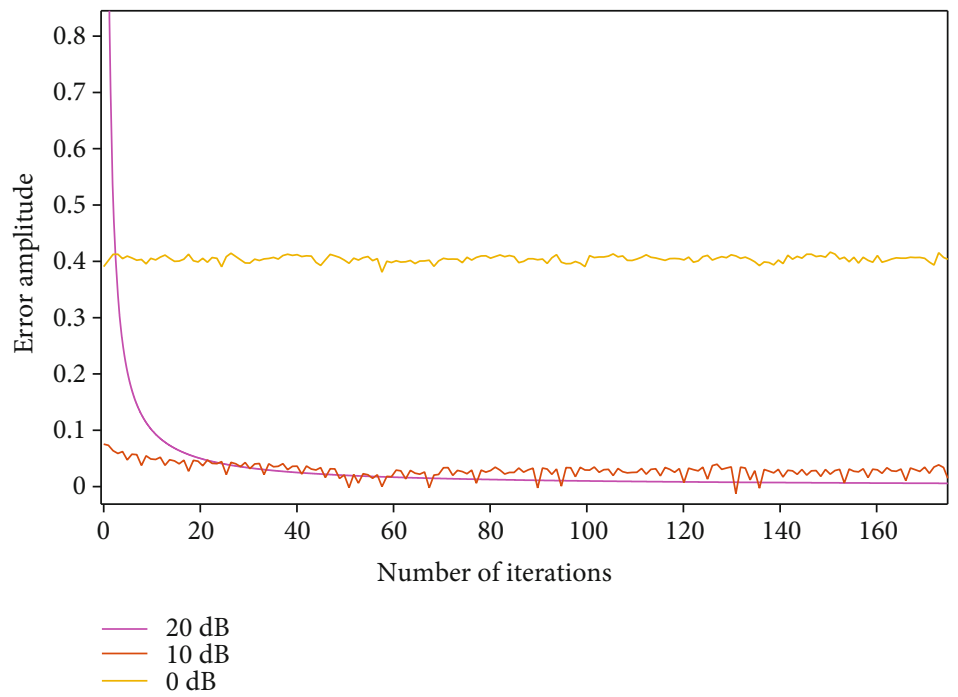

Figure 5: Error convergence.

The signal-to-interference noise ratio can be obtained from (15) to (17):

$$
\operatorname{SINR}=P_{\text {expectation }}\left(P_{\text {interfere }}+P_{\text {noise }}\right)^{-1} .
$$

According to the signal-to-interference noise ratio, the expression of spectral efficiency can be obtained as follows:

$$
\mathrm{SE}=\log _{2}|1+\mathrm{SINR}| \text {. }
$$

In the experiment, make the step size $\mu=0.0001$. The output of the first stage is used as the input of the LMS adaptive algorithm. After the algorithm is executed, the convergence of the error during the algorithm execution can be observed through Figure 5. It shows that as the signal-tonoise ratio (SNR) increases, the error convergence accelerates; When $\mathrm{SNR}=20 \mathrm{~dB}, 50$ iterations can achieve convergence. The classification accuracy expresses the similarity between the test set and the classification set. The more similar the classification accuracy is, the closer it is to $100 \%$, indicating that the more stable the whole system is, the lower the energy level is. Experiments show that the smaller the convergence error is, the closer the classification accuracy is to $100 \%$. That is, the longer the time, the less energy.

The signal-to-interference noise ratio and spectral efficiency are shown in Figures 6(a) and 6(b), respectively. It can be seen that the signal-to-interference noise ratio and spectral efficiency increase with the increase of signal-tonoise ratio. The SNR and spectral efficiency of the proposed hybrid beamforming scheme are much higher than that of pure analog beamforming and close to that of pure digital beamforming.

4.2. Simulation Results and Analysis. In the simulation environment, the settings are described as follows: the number of transmitting antennas of the base station $N_{t}=128$, the number of receiving antennas of each user $N_{r}=32$, the number of users $k=4$, the transmission angle and arrival angle are evenly distributed within $[0,2 \pi]$, and the antenna interval of the base station $D=\lambda / 2$. The data stream of each user is $S_{r}=4$, the data stream of the base station is $s t=K S_{r}$, the total transmission power is $P=K$ $S_{r}$, and the scattering path between the base station and each user is $L K=8$.

Figure 7 shows the speed and performance comparison between the block diagonal beamforming algorithm based on singular value decomposition and the proposed algorithm based on approximate inversion of Neumann series for complete digital beamforming. As can be seen from Figure 7, the sum rate of this algorithm is close to the algorithm based on singular value decomposition. Because the approximate inversion based on Neumann series is used instead of singular value decomposition, it has lower computational complexity than the algorithm based on singular value decomposition [22].

Figure 8 shows the user and rate conversion curve when the number of RF links is 3-8 under the signal-to-noise ratio $R_{\mathrm{SN}}=0$ and $R_{\mathrm{SN}}=10 \mathrm{~dB}$. As can be seen from Figure 8, with the increase of the number of RF links, the user and rate of this algorithm continue to approach complete digital beamforming, and the performance of this algorithm is basically the same as that of the MO algorithm [23].

4.3. Relationship between Optimal Energy Efficiency $\eta_{E E}{ }^{*}$ and $N$ of Sensor. Given the spectral efficiency (SE), whether $N$ $M=L$ or $N$ and $M$ are independent of each other, there is only one optimal number of RF links $N *$ to obtain the best energy efficiency (EE). According to the existing analysis, it is concluded that when only the changes of energy efficiency (EE) and spectral efficiency (SE) are considered, there is only one green point on the EE-SE curve. At the same time, for a given spectral effect (SE), the optimal number of RF links $N$ is only one. However, the relationship between the optimal energy efficiency point $\eta_{\mathrm{EE}}{ }^{*}$ and the number of RF links $N$ is not clear. When the number of RF links $N$ changes, whether the optimal energy efficiency point $\eta_{\mathrm{EE}}{ }^{*}$ obtained 


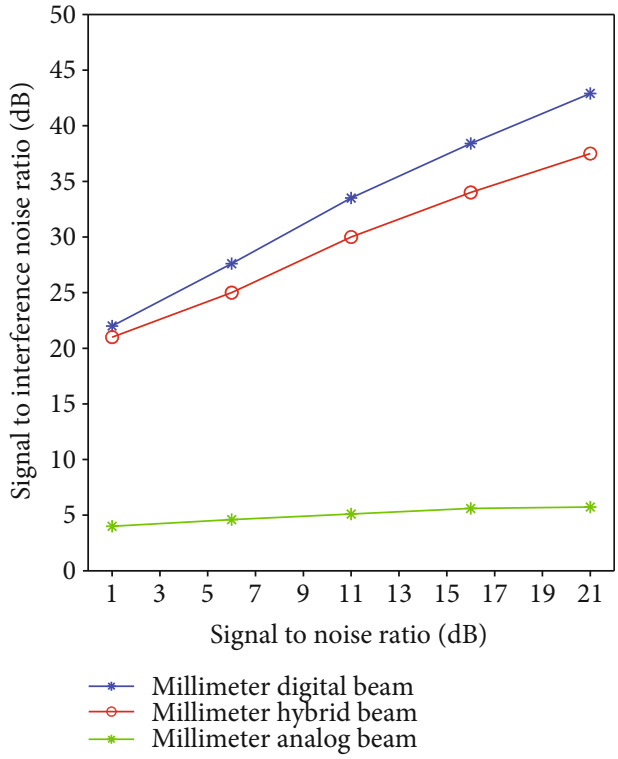

(a)

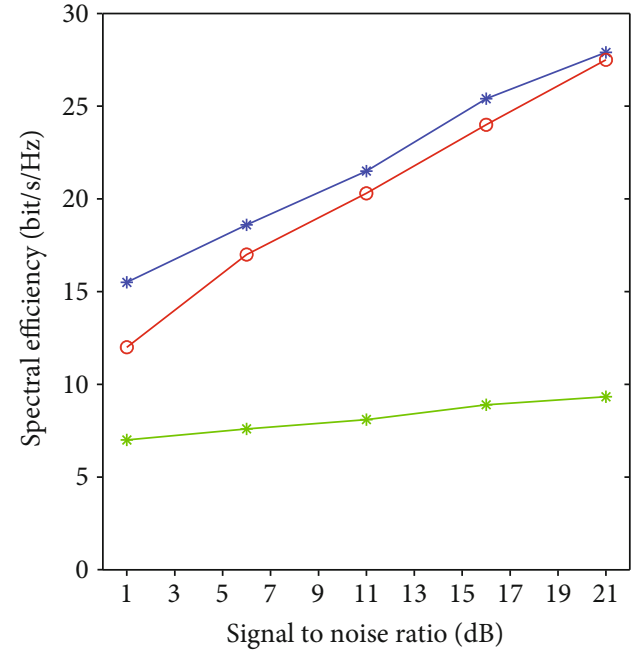

(b)

Figure 6: (a) Signal-to-interference noise ratio simulation results. (b) Spectrum efficiency simulation results.

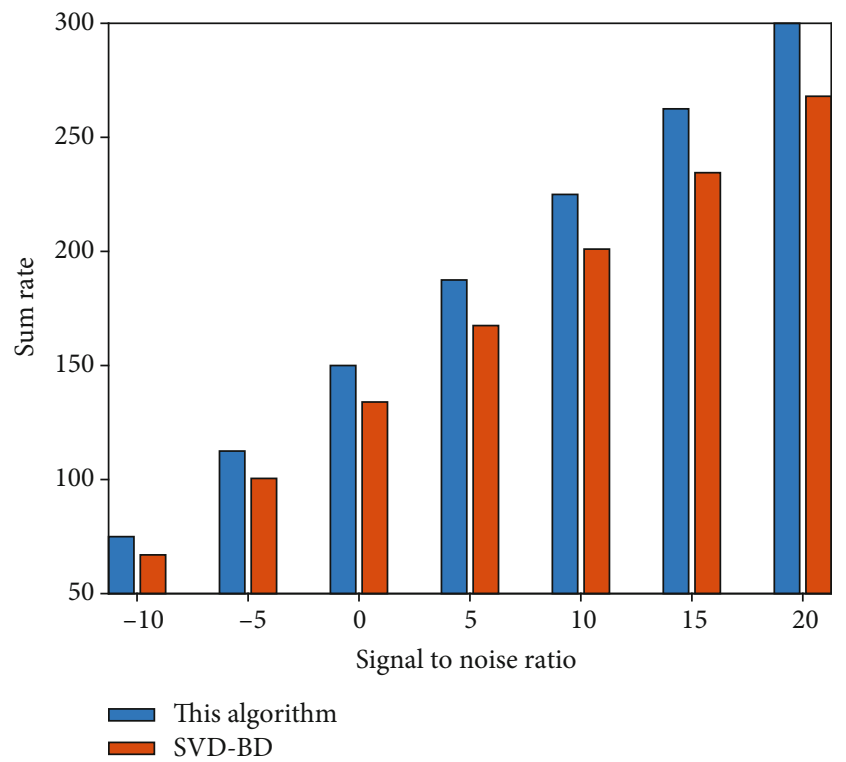

FIGURE 7: Rate comparison of two complete digital beamforming algorithms.

at the green point is really the optimal energy efficiency that can be obtained.

When the spectral efficiency (SE) is given, the impact of the number of digital links $N$ on energy efficiency is shown in Figure 9.

Because $N$ and $M$ must be integers, only $(1,200),(2,100)$, $(4,50),(5,40),(8,25)$, and $(10,20)$ are valid combinations of $(N, M)$ in the graph with $N M=200$. We can see the following:

(i) On each curve, there is an $N$ value that can get the highest energy efficiency (EE). For example, when

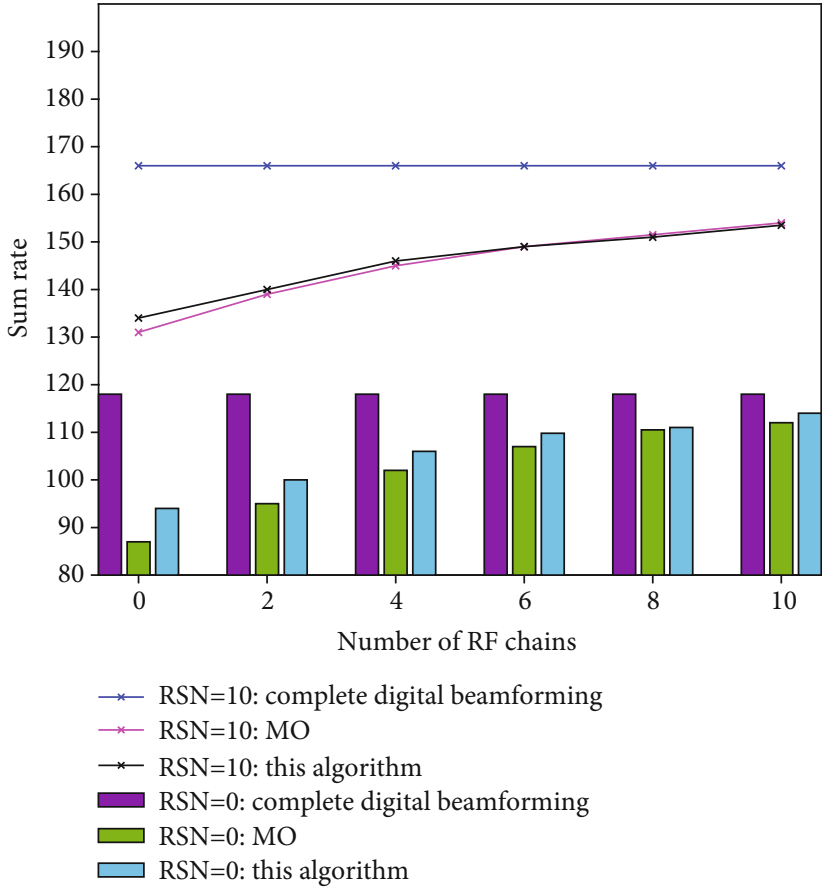

Figure 8: Sum rate curves under different RF chains.

the value of $S E$ is $20 \mathrm{~b} / \mathrm{s} / \mathrm{Hz}$, the best $N=4$; when the value of $S E$ is $12 \mathrm{~b} / \mathrm{s} / \mathrm{Hz}$, the best $N=2$

(ii) When the value of $N$ is small, the energy efficiency (EE) is more sensitive to the change of $N$. When the value of $N$ increases, the value of energy efficiency (EE) is almost unchanged

When $N$ and $M$ are independent of each other, the effect of $N$ on energy efficiency (EE) is shown in the right figure in 


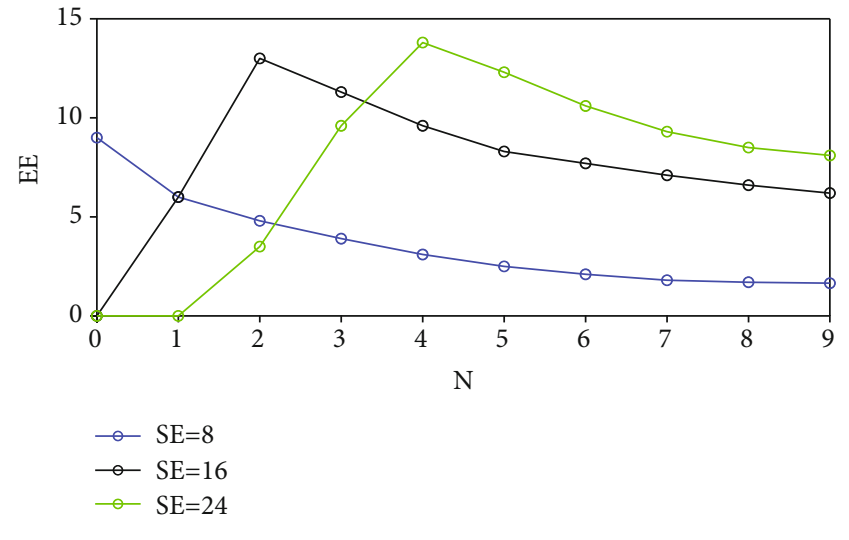

Figure 9: Relationship between $N$ and EE.

Figure 9. Where $M=40$, other parameters remain unchanged. Similar to the case of $N M=200$, there is an optimal $N$ value on each curve. For example, when the value of SE is $40 \mathrm{~b} / \mathrm{s} / \mathrm{Hz}$, the best $N=5$; when the value of $S E$ is $16 \mathrm{~b} / \mathrm{s} / \mathrm{Hz}$, the best $N=2$. In particular, when $N$ and $M$ are independent of each other, the energy efficiency (EE) is very sensitive to $N$, and the energy efficiency (EE) first increases and then decreases with the change of $N$ value.

\section{Conclusion}

This paper analyzes the training process of millimeter hybrid beamforming for $5 \mathrm{G}$ and analyzes the antenna beam state of the transceiver in each stage. Then, the link level simulation of $802.11 \mathrm{ad}$ is carried out, the simulation platform is built, each module of the platform is described in detail, and the simulation curve is given for calibration. At the same time, the digital domain beamforming is verified. Then, through the study of a cascaded hybrid beamforming architecture, the effects of RF link number $N$ and antenna number $N M$ on the performance of millimeter-wave wireless communication system are analyzed, including spectral efficiency (SE) and energy efficiency (EE). Firstly, the relationship between energy efficiency (EE), spectral efficiency (SE), and $\mathrm{RF}$ link number $N$ under partial connection architecture is analyzed. It can be proved that there is an optimal power point, namely, green point, on the premise of considering the link device power. Secondly, the influence of link number $N$ on energy efficiency under the condition of given spectral efficiency (SE) is analyzed. The analysis shows that when the spectral efficiency (SE) is determined, there is an optimal RF link number $N^{*}$ which can make the system obtain the best energy efficiency (EE). Finally, the optimal energy efficiency is analyzed $\eta$. The results show that when the number of RF links $N$ is not related to the number of antennas $M$ in each subarray, $\eta_{\mathrm{EE}}{ }^{*}$ increases monotonically with $N$; when $N M=L$, the optimal energy efficiency (EE) can be obtained only when $N$ meets certain conditions. The conclusion of these relations can be used to guide the design of millimeter-wave communication hybrid beamforming architecture to obtain better energy efficiency.
Compared with the traditional hybrid beamforming technology, which needs high complexity operations such as high-dimensional channel estimation and SVD decomposition, this paper combines beam scanning and adaptive algorithm to avoid high complexity operations such as channel estimation and SVD decomposition. In the first stage, the analog domain beam scanning obtains the beam closest to the desired user direction; the adaptive algorithm in the second stage fine tunes the beam obtained in the first stage. The simulation results show that when the number of RF links is only 16 , the beam pattern obtained by hybrid beamforming can accurately point to the desired user and produce a deep "null" in the direction of interfering users. In terms of signalto-interference noise ratio and spectral efficiency, the proposed hybrid beamforming scheme can well approach pure digital beamforming.

\section{Data Availability}

The data used to support the findings of this study are available from the corresponding author upon request.

\section{Conflicts of Interest}

The author declares that there are no known competing financial interests or personal relationships that could have appeared to influence the work reported in this paper.

\section{References}

[1] A. Shahmansoori, G. E. Garcia, G. Destino, G. Seco-Granados, and $\mathrm{H}$. Wymeersch, "Position and orientation estimation through millimeter-wave MIMO in 5G systems," IEEE Transactions on Wireless Communications, vol. 17, no. 3, pp. 1822$1835,2018$.

[2] K. Shafique, B. A. Khawaja, F. Sabir, S. Qazi, and M. Mustaqim, "Internet of things (IoT) for next-generation smart systems: a review of current challenges, future trends and prospects for emerging 5G-IoT scenarios," Ieee Access, vol. 8, pp. 23022 23040, 2020.

[3] R. W. Heath, N. Gonzalez-Prelcic, S. Rangan, W. Roh, and A. M. Sayeed, "An overview of signal processing techniques for millimeter wave MIMO systems," IEEE Journal of Selected Topics in Signal Processing, vol. 10, no. 3, pp. 436-453, 2016.

[4] V. Raghavan, A. Partyka, A. Sampath et al., "Millimeter-wave MIMO prototype: measurements and experimental results," IEEE Communications Magazine, vol. 56, no. 1, pp. 202-209, 2018.

[5] R. Chataut and R. Akl, "Massive MIMO systems for 5G and beyond networks-overview, recent trends, challenges, and future research direction," Sensors, vol. 20, no. 10, p. 2753, 2020.

[6] T. Kim, J. Park, J.-Y. Seol, S. Jeong, J. Cho, and W. Roh, “Tens of Gbps support with mmWave beamforming systems for next generation communications," in 2013 IEEE Global Communications Conference (GLOBECOM), pp. 3685-3690, Atlanta, GA, USA, 2013.

[7] R. A. Stirling-Gallacher and M. S. Rahman, "Multi-user MIMO strategies for a millimeter wave communication system using hybrid beam-forming," in 2015 IEEE International 
Conference on Communications (ICC), pp. 2437-2443, London, UK, 2015.

[8] L. Liang, W. Xu, and X. Dong, "Low-complexity hybrid precoding in massive multiuser MIMO systems," IEEE Wireless Communications Letters, vol. 3, no. 6, pp. 653-656, 2014.

[9] O. El Ayach, S. Rajagopal, S. Abu-Surra, Z. Pi, and R. W. Heath, "Spatially sparse precoding in millimeter wave MIMO systems," IEEE Transactions on Wireless Communications, vol. 13, no. 3, pp. 1499-1513, 2014.

[10] J. Singh and S. Ramakrishna, "On the feasibility of codebookbased beamforming in millimeter wave systems with multiple antenna arrays," IEEE Transactions on Wireless Communications, vol. 14, no. 5, pp. 2670-2683, 2015.

[11] F. Sohrabi and W. Yu, "Hybrid analog and digital beamforming for mmWave OFDM large-scale antenna arrays," IEEE Journal on Selected Areas in Communications, vol. 35, no. 7, pp. 1432-1443, 2017.

[12] J. C. Chen, "Hybrid beamforming with discrete phase shifters for millimeter-wave massive MIMO systems," IEEE Transactions on Vehicular Technology, vol. 66, no. 8, pp. 7604-7608, 2017.

[13] J. Zhang, Y. Huang, Q. Shi, J. Wang, and L. Yang, “Codebook design for beam alignment in millimeter wave communication systems," IEEE Transactions on Communications, vol. 65, no. 11, pp. 4980-4995, 2017.

[14] M. Rihan and L. Huang, "Optimum co-design of spectrum sharing between MIMO radar and MIMO communication systems: an interference alignment approach," IEEE Transactions on Vehicular Technology, vol. 67, no. 12, pp. 1166711680, 2018.

[15] J. Cong, T. Lin, and Y. Zhu, "Hybrid MMSE beamforming for multiuser millimeter-wave communication systems," IEEE Communications Letters, vol. 22, no. 11, pp. 2390-2393, 2018.

[16] Q. Shi and M. Hong, "Spectral efficiency optimization for millimeter wave multiuser MIMO systems," IEEE Journal of Selected Topics in Signal Processing, vol. 12, no. 3, pp. 455468, 2018.

[17] I. Ahmed, H. Khammari, and A. Shahid, "Resource allocation for transmit hybrid beamforming in decoupled millimeter wave multiuser-MIMO downlink," IEEE Access, vol. 5, pp. 170-182, 2017.

[18] S. S. Ioushua and Y. C. Eldar, "A family of hybrid analog-digital beamforming methods for massive MIMO systems," IEEE Transactions on Signal Processing, vol. 67, no. 12, pp. 32433257, 2019.

[19] K. Roth, H. Pirzadeh, A. L. Swindlehurst, and J. A. Nossek, “A comparison of hybrid beamforming and digital beamforming with low-resolution ADCs for multiple users and imperfect CSI," IEEE Journal of Selected Topics in Signal Processing, vol. 12, no. 3, pp. 484-498, 2018.

[20] D. Sikri and R. M. Jayasuriya, "Multi-beam phased array with full digital beamforming for SATCOM and 5G," 5G Phased Array Technologies, 2019.

[21] B. di, H. Zhang, L. Li, L. Song, Y. Li, and Z. Han, "Practical hybrid beamforming with finite-resolution phase shifters for reconfigurable intelligent surface based multi-user communications," IEEE Transactions on Vehicular Technology, vol. 69, no. 4, pp. 4565-4570, 2020.
[22] Z. C. Li, M. G. Lee, H. T. Huang, and J. Y. Chiang, "Neumann problems of 2D Laplace's equation by method of fundamental solutions," Applied Numerical Mathematics, vol. 119, pp. 126$145,2017$.

[23] L. Sanguinetti, E. Björnson, and J. Hoydis, "Toward massive MIMO 2.0: understanding spatial correlation, interference suppression, and pilot contamination," IEEE Transactions on Communications, vol. 68, no. 1, pp. 232-257, 2020. 